\title{
Plaidoyer pour une orthodontie précoce
}

\section{RÉSUMÉ}

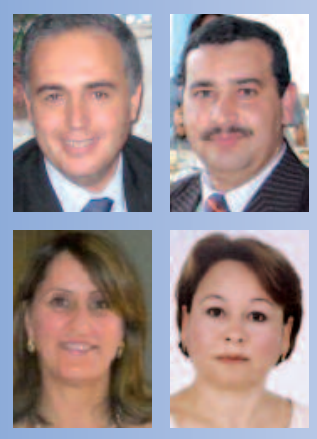

Adel BEN AMOR

Professeur hospitalo-universitaire en orthodontie,

Responsable pédagogique,

de l'enseignement de I'ODF,

Chef du service d'orthopédie dento-faciale

à la clinique odontologique

hospitalo-universitaire de Monastir,

Faculté de médecine dentaire

Université de Monastir,

Avenue Avicenne 5019,

Monastir, Tunisie.

\section{Samir TOBJI}

Professeur agrégé en orthodontie, Responsable de l'enseignement d'ODF : Institut supérieur de sciences et techniques de la santé de Monastir,

Faculté de médecine dentaire,

Université de Monastir,

Tunisie.

\section{Anissa ZINELABIDINE}

Professeur agrégée

en orthopédie dento-faciale,

Faculté de médecine dentaire,

Université de Monastir,

Tunisie.

\section{Faten BEN AMOR}

Chef de service des consultations externes à la clinique hospitalo-universitaire de médecine dentaire de Monastir. Responsable du laboratoire d'anatomie,

Faculté de médecine dentaire

Université de Monastir,

Tunisie.
L'approche précoce des malocclusions dentaires mériterait plus d'intérêt de la part de notre profession. Devant la recrudescence des problèmes fonctionnels et leurs implications dans l'installation des décalages squelettiques, l'orthodontiste a le devoir de maitriser toutes les connaissances nouvelles en matière d'interception qui lui permettront un jour d'agir le plus tôt possible, en toutes circonstances, pour le plus grand bien des patients. 


\section{Introduction}

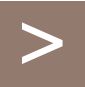

Depuis quelque temps déjà, les thérapeutiques orthodontiques constituent I'une de nos préoccupations en matière de santé. En effet, nous pouvons considérer les malocclusions dentaires comme des maladies présentant des tableaux cliniques dont les étiologies peu- vent être maîtrisées. Il serait donc intéressant de pouvoir prévenir et surtout intercepter celles-ci avant leur installation. Nous proposons de faire le point sur l'orthodontie précoce en présentant les justificatifs de cette approche et les moyens que nous pouvons mettre en œuvre.

\section{Justificatifs de l'approche précoce}

L'idée la plus répandue en orthodontie est qu'il vaut mieux attendre l'évolution des dents définitives vers 12 ans pour traiter les malocclusions avec l'outil multibagues qui donne d'excellents résultats.

Or, si l'orthodontiste " attend ", il laissera les problèmes fonctionnels, alvéolaires et squelettiques s'installer, au risque de se retrouver devant des cas ortho-chirurgicaux qui auraient pu être évités par un abord précoce auquel nous sommes tous sensibles aujourd'hui. "Nous traitons aujourd'hui les enfants des parents que nous avons opérés hier " Vesse [1].

Si le caractère génétique et héréditaire des malocclusions tarde aujourd'hui à être maîtrisé, il n'en demeure pas moins que nous pouvons agir précocement sur les phénomènes d'éruption dentaire et surtout sur les dysfonctions de la langue, des lèvres et des joues lors de la déglutition, phonation, mastication et respiration engendrant des rattrapages des décalages squelettiques et alvéolaires dans les trois sens de l'espace.

Nous n'insisterons jamais assez sur les dégâts que pourra occasionner par exemple une position basse et protrusive de la langue sur la position des dents et des maxillaires (fig.1). De plus, ne pas traiter suffisamment tôt une malocclusion décelée est une non-assistance à personne en danger, et une perte de chance pour les
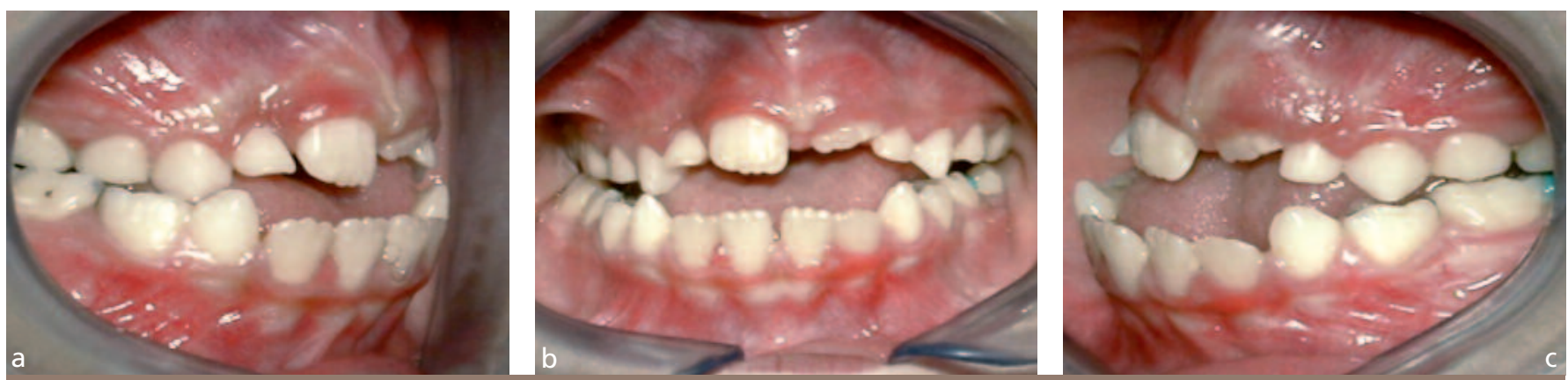

Fig. 1 a à c Vues d'une béance alvéolaire antérieure suite à une déglutition dysfonctionnelle chez un patient de 7 ans et demi. 
patients, Béry [2] y voit un aspect médico-légal : " la responsabilité du praticien est engagée en cas de non-information donnée au patient ou de non-intervention précoce laissant s'aggraver sa dysmorphose corrigible ".

Force est de constater aujourd'hui que les thérapeutiques précoces donnent d'excellents résultats, connus et reconnus à travers les publications et les conférences internationales [3-9].
Cependant leur maîtrise dépend fortement de la volonté des orthodontistes à favoriser et à propager leur enseignement de sorte que tout médecin dentiste puisse avoir accès à ce savoir.

Le monde n'est-il pas aujourd'hui un village ouvert grâce aux procédés de communication et même nos patients peuvent comprendre les techniques orthodontiques?

\section{Possibilités thérapeutiques}

Interception

des problèmes d'éruption et de leurs conséquences

S'il est un volet où l'interception est délicate, c'est bien l'interception de l'encombrement dentaire car elle est souvent imprévisible. En effet, la DDM peut être transitoire et résulter d'un problème de croissance tardive des arcades dentaires tout comme elle peut s'aggraver d'un coup avec des phénomènes d'ectopie dentaire et d'inclusion canine.
Une bonne lecture des radiographies panoramiques et une bonne connaissance des phénomènes de croissance sont nécessaires. Si des actes tels que les guidances d'éruptions par des extractions bien pensées dans le temps, par des meulages sélectifs et surtout des mainteneurs d'espace, des lip-bumpers et des pare-chocs labiaux sont recommandés (fig. 2), les extractions pilotées doivent être délaissées vu l'engagement total que nécessitent ces interventions de la part du patient et du praticien, avec des conséquences graves en cas d'erreur (fig. 3).
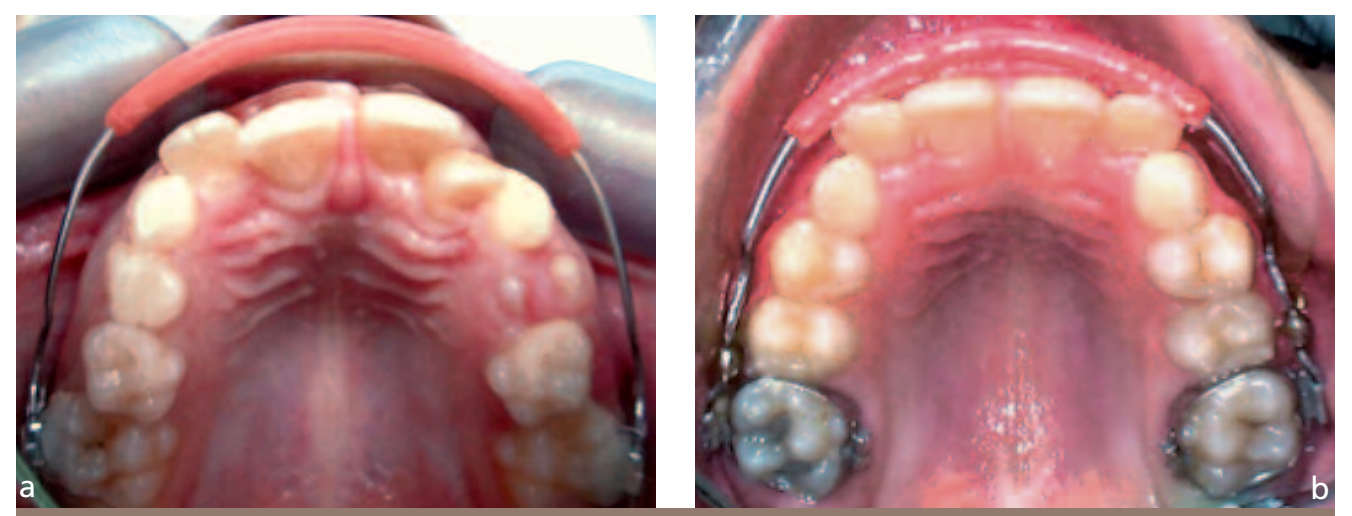

Fig. 2 a et $b$

a : lip-bumper mis à l'arcade maxillaire ;

$\mathrm{b}$ : résultat après 9 mois. 


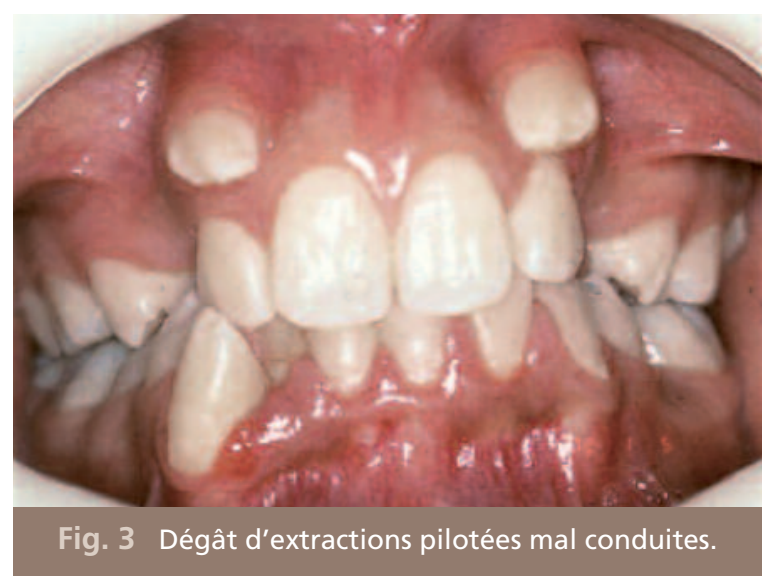

\section{Sens antéropostérieur}

Les thérapeutiques orthodontiques du sens antéropostérieur ont fait leurs preuves, qu'il s'agisse d'activateurs de classe II ou des masques faciaux pour la classe III. Toutefois, la réussite de ces thérapeutiques est tributaire d'une maîtrise de leurs indications et contreindications et surtout de la biomécanique permettant le rattrapage des décalages squelettiques. Il ne faut pas oublier que ces malocclusions ont souvent une étiologie fonctionnelle dont la prise en charge doit être faite en parallèle.
Cependant, le contrôle du sens vertical demeure la clef de la stabilité des résultats obtenus et un deuxième temps de traitement en multibagues est indispensable pour parfaire le résultat.

\section{Sens vertical}

Nous évoquerons deux problèmes récurrents en orthodontie, à savoir la béance dentaire et la supraclusion qui nécessitent une prise en charge précoce.

En effet, la béance dentaire a toujours une étiologie qui nécessite une rééducation et nous pro-

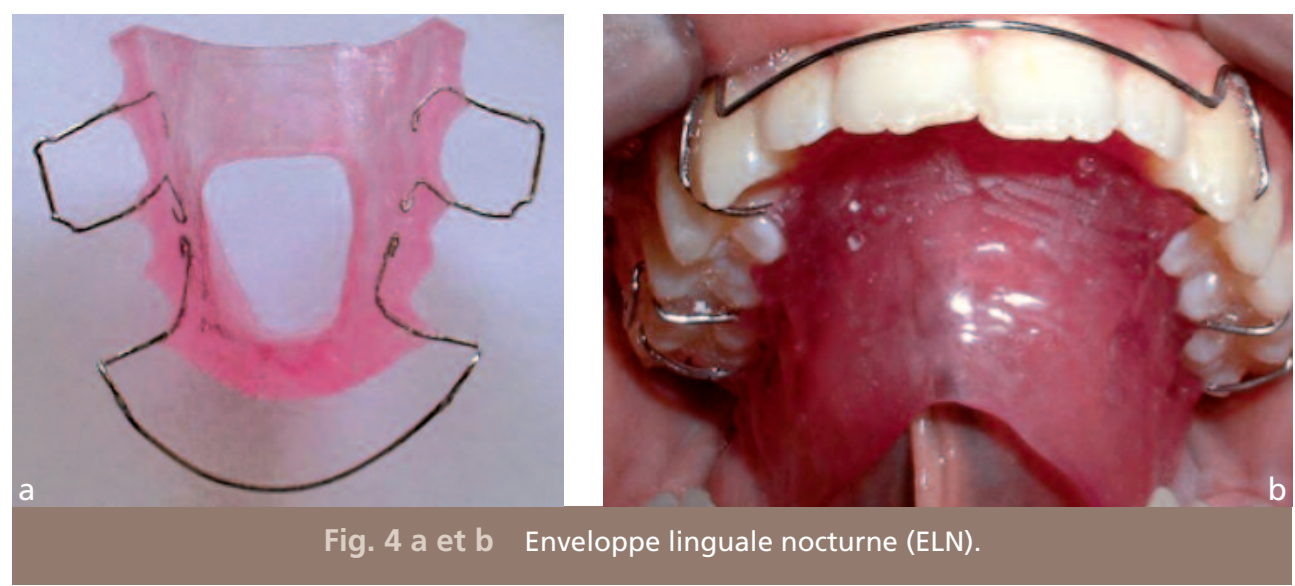


posons toujours en priorité l'enveloppe linguale nocturne (ELN) (fig. 4) qui donne d'excellents résultats. Cet ascenseur permet à la langue d'acquérir de nouvelles habitudes de positionnement, particulièrement en cas de déglutition dysfonctionnelle. II faut toujours rechercher une stabilité de l'engrènement dentaire qui fermera la porte à toute forme de récidive de la malocclusion.

Pour les patients peu motivés pour le port d'appareillage amovible encombrant, la grille antilangue sur bague est une alternative intéressante.

Toutefois, un suivi radiologique de la croissance est nécessaire pour voir l'évolution du sens vertical et les superpositions structurales sont un excellent exercice.

D'un autre côté, la supraclusion doit nous interpeller dès la denture temporaire et nous devons prévoir d'agir lors de l'évolution des incisives définitives, par des cales antérieures ou en agissant sur l'égression des molaires. Il faut avoir à l'esprit que le fait de libérer les incisives des pressions de l'orbiculaire a une action sur le redressement des dents.

\section{Sens transversal}

La stratégie thérapeutique du sens transversal dépend en premier de notre capacité à établir un diagnostic différentiel entre l'endoalvéolie et l'endognathie.

Si le problème est squelettique, nous préconisons d'effectuer une disjonction rapide du maxillaire par un disjoncteur type Hyrax $^{\circledR}$ (fig. 5), ceci avant la solidarisation de la suture palatine médiane.

Si le problème est alvéolaire, nous pouvons espérer une amélioration de la forme d'arcade en posant un quadhélix qui permettra d'assurer un bon agencement des dents.

Vers l'âge de 4 ans et si en plus d'un inversé d'articulé latéral nous avons une latérodéviation associée qui pourra évoluer vers l'asymétrie basale, nous pouvons opérer des meulages sélectifs progressifs afin de supprimer les interférences. Si le problème est plus important, on peut utiliser une plaque à vérin avec plan de surélévation et plan de guidage (fig. 6). Il s'agit souvent d'un problème de mastication et toutes

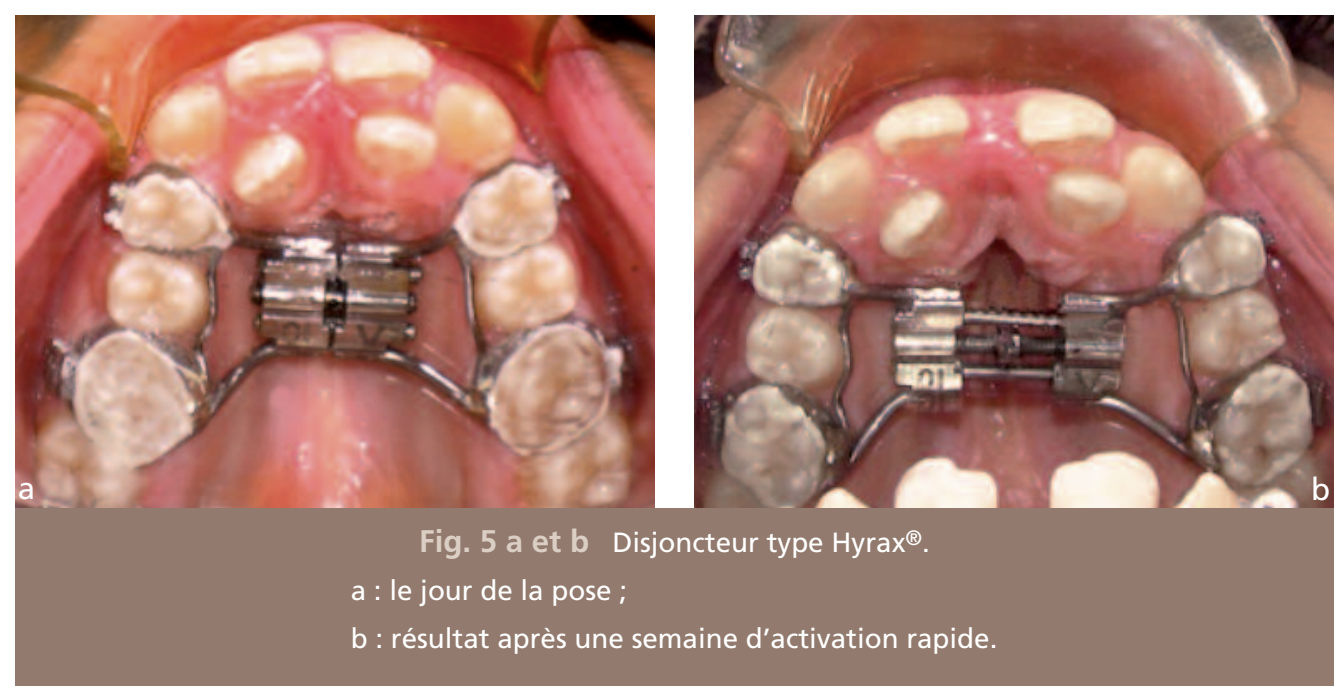



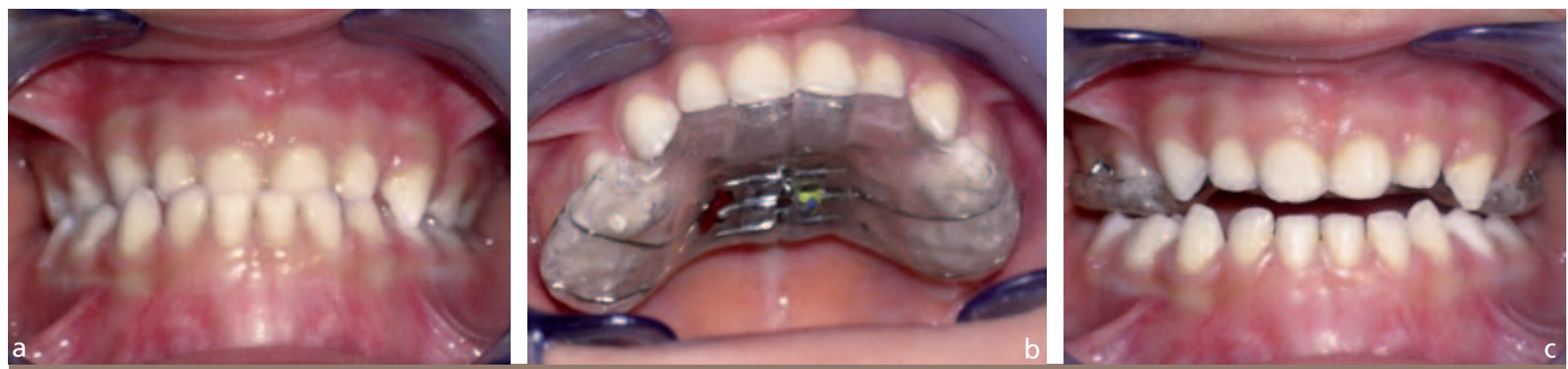

Fig. 6 a à c Plaque à vérin médian avec plan de surélévation et de guidage chez une patiente âgée de 4 ans présentant une latérodéviation mandibulaire.

les théories de Planas sont fabuleusement enrichissantes dans ce domaine.

\section{Chirurgie \\ pré-orthodontique}

Le médecin dentiste doit être sensible aux problèmes de végétations adénoïdes et d'amygdales hypertrophiques qui peuvent provoquer une obstruction des voies aériennes supérieures. Il doit jouer pleinement son rôle de véritable médecin de famille et sauver ces enfants de la respiration buccale et de ses conséquences qui prédisposent au syndrome de I'apnée du sommeil (SAHOS) qui nous préoccupe tant aujourd'hui [10].

Un autre volet chirurgical concerne l'insertion des freins de la langue et des lèvres (fig. 7). Une brièveté du frein lingual entraînera inéluctablement une langue basse et antérieure, la section de ce frein libérera la langue des troubles fonctionnels et une rééducation du tonus lingual permettra de retrouver l'équilibre labio-linguo-jugal tant recherché.

Nous avons conçu un tonificateur ou « booster lingual » dans le service d'orthodontie de
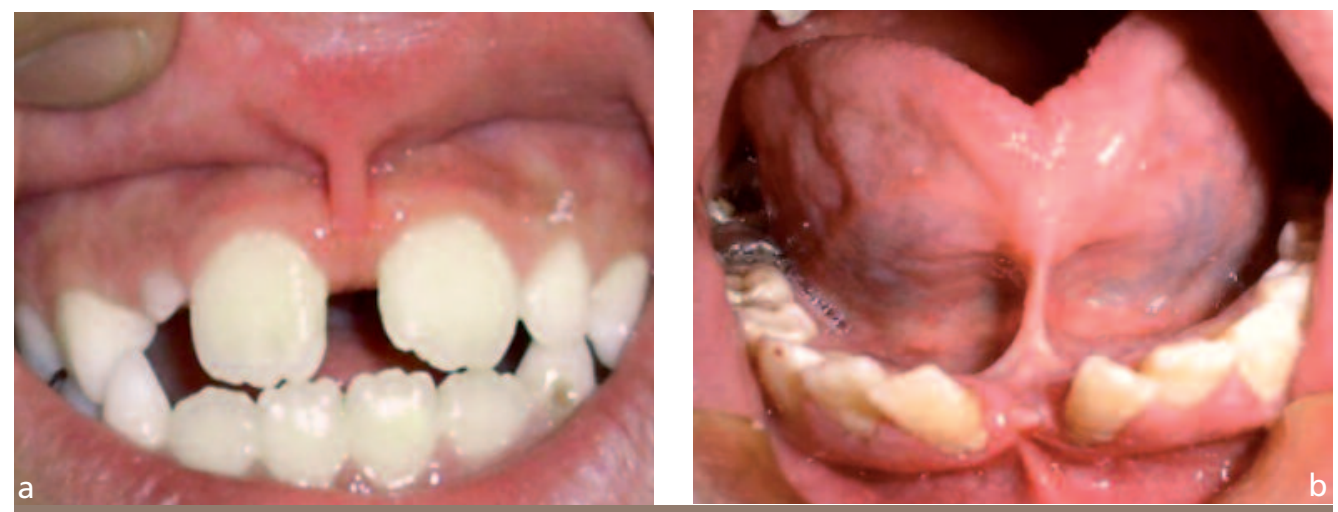

Fig. 7 a et b

a : frein labial supérieur court et fibreux ;

b : brièveté du frein lingual. 
Monastir qui agit de façon ludique et qui donne des effets immédiats (fig. 8).

En cas de frein labial large et fibreux gênant les dents et provoquant un blanchiment lors de l'étirement de la lèvre, nous devons indiquer sa résection à seule fin de permettre une succession normale de l'éruption dentaire. Delaire [11] préconise globalement de le faire avant l'éruption complète des incisives latérales et nous sommes d'accord avec cette théorie bien qu'elle soit controversée.

D'autre part, le médecin dentiste doit être attentif à la présence des dents surnuméraires qui pourra perturber l'occlusion. Dans tous les cas, l'orthodontie ne peut se pratiquer que dans un esprit cartésien et médical ouvert sur toutes les spécialités et avec une projection sur l'avenir des patients.
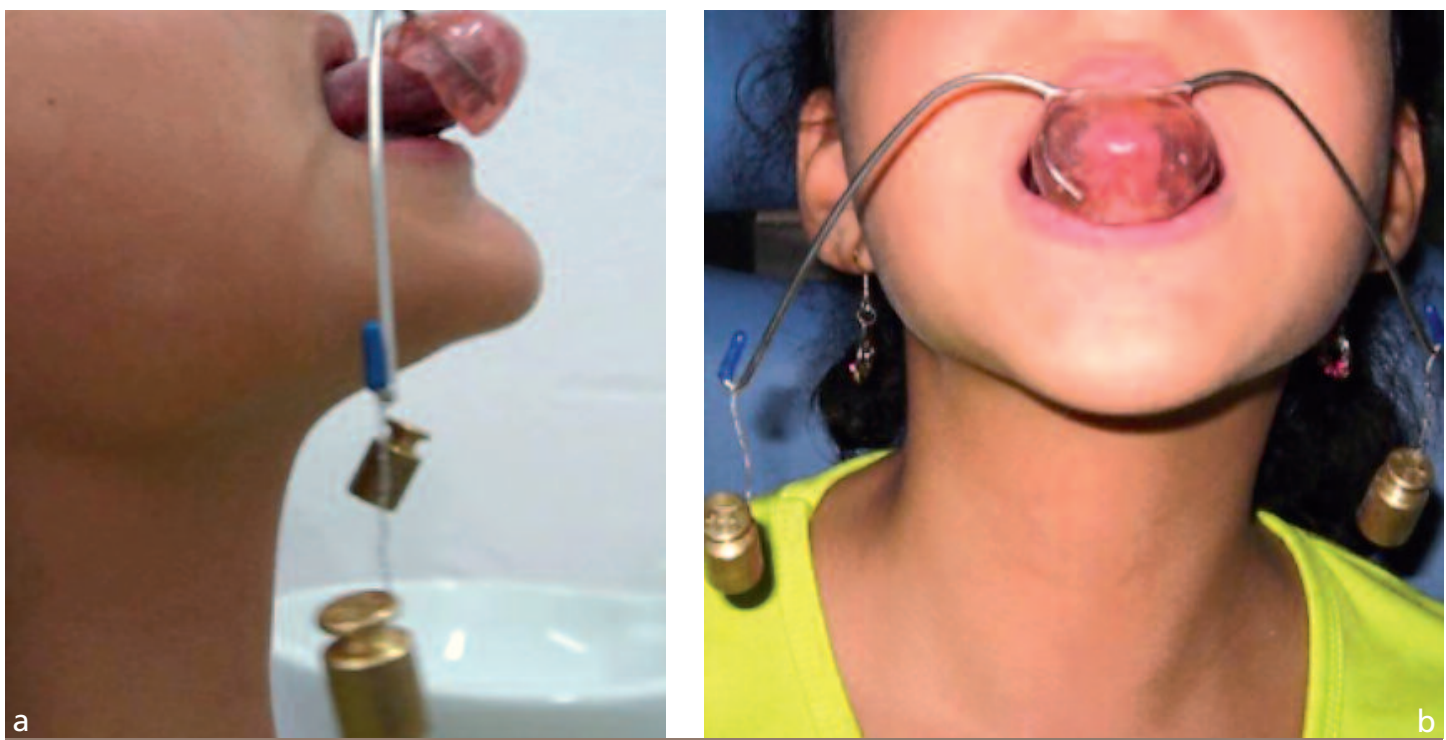

Fig. 8 a et b Booster lingual.

\section{Conclusion}

La pratique de l'orthodontie précoce nécessite des connaissances poussées en céphalométrie, croissance, biomécanique, sémiologie, médecine et chirurgie buccales, ORL., maxillo-faciale, psychologie, ce qui fait que sa maîtrise est un art.

Il faut rester attentif et vigilant quant à la bibliographie qui laisserait suggérer parfois qu'il n'y a " aucune différence significative entre un traitement orthodontique réalisé en un temps et en deux temps au niveau des résultats " [12] et que par conséquent il vaut mieux attendre le multibagues.

Nous devons militer pour que cette orthodontie médicalisée soit la véritable orthodontie car " pour agir il faut d'abord comprendre ». 


\section{Bibliographie}

1. Vesse M.

\section{Arguments}

à propos de la chirurgie des fonctions oro-faciales. Orthod Fr 1998;69(1):205-6.

2. Béry $A$.

En l'absence de traitement d'orthodontie précoce, est-il une perte de chance ?. Ortho Fr 2006;77(2):327-33.

3. McNamara JA Jr, Brudon WL. Orthodontic and orthopedic treatment in the mixed dentition. Ann Arbor: Needham Press, 1993:3-6.

4. Viazis $A D$.

Efficient orthodontic treatment timing. Am J Orthod Dentofacial Orthop 1995;108:560-1.

5. Tulloch JFC, Phillips $C$, Koch G, Proffit WR.

The effect of early intervention on skeletal pattern in Class II molocclusion: a randomized clinical trial. Am J Orthod Dentofacial Orthop 1997;111:391-400.

6. O'Brien K, Wright J, Conboy F,
SanjieY, Mandall N, Chadwick S, Connolly I, Cook P, Birnie D, Hammond M, Harradine N, Lewis D, McDade C, Mitchell L, Murray A, O'Neill J, Read M, Robinson S, Roberts-Harry D, Sandler J, Shaw I.

Effectiveness of early orthodontic treatment with the twin-block appliance: A multicenter, randomized, controlled trial. Part 1: Dental and skeletal effects.

Am J Orthod Dentofacial Orthop 2003;124:488-94.

7. Weyant R.

Early orthodontic treatment is no more effective in treating prominent upper front teeth (Class II malocclusion) than late treatment. J Evid Based Dent Pract 2008;8(2):72-3.

8. Gregory JK, Pongsri B. Effectiveness of interceptive orthodontic treatment in reducing malocclusions.
Am J Orthod Dentofacial Orthop 2010;137:18-25.

9. Fellus $P$..

Orthodontie précoce en dentaire temporaire Paris : CdP, 2003

10. Yongming $\mathrm{L}$. Early orthodontic treatment of skeletal Class II malocclusiuon may be effective to prevent the potential for OSAHS and snoring. Med Hypotheses 2009;73(4):594-5.

11. Delaire J, Mercier J, Gordeeff A, Bedhet N. The 3 palatine fibromucous membranes. Their role in maxillary growth. Therapeutic role in surgery of the palatine shelves. Rev Stomatol Chir Maxillofac 1989;90(6):379-90.

12. Gianelly AA.

One-phase versus two-phase treatment. Am J Orthod Dentofacial Orthop 1995; 108:556-9.

\section{SUMMARY}

\section{Plea for early treatment in orthodontics}

Adel BEN AMOR, Samir TOBJI, Anissa ZINELABIDINE, Faten BEN AMOR

\section{Keywords \\ - interception \\ - orofacial function \\ - activator \\ - early orthodontics}

The early approach of dental malocclusion deserves more interest from our profession. Before the rise of functional problems and their involvement in the installation of skeletal discrepancies, the orthodontist has the duty to master all the new knowledge for interception which will one day permit him to act as soon as possible in all circumstances for the good of our patients. 